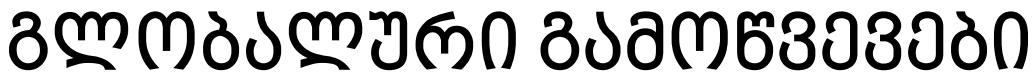

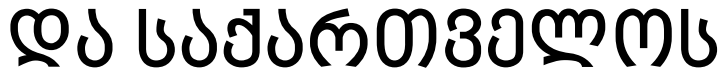

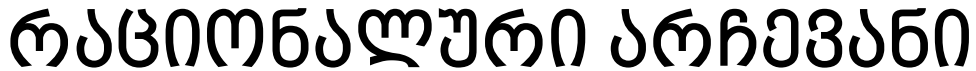

https://doi.org/10.35945/gb.2016.02.001

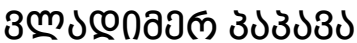

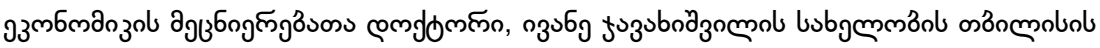

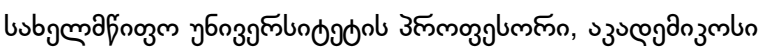

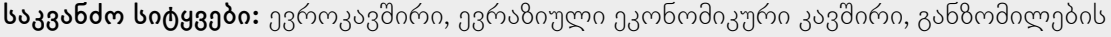
ठumngुno

2015

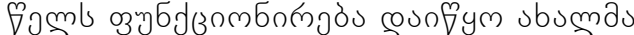

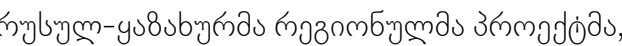

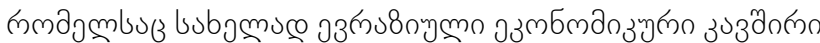

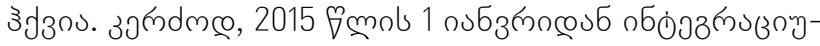

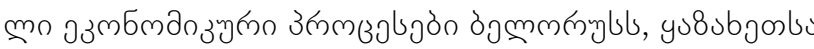

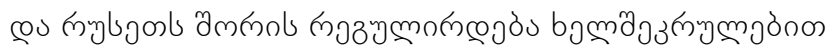

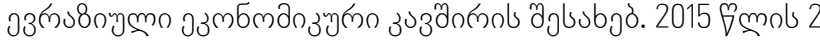

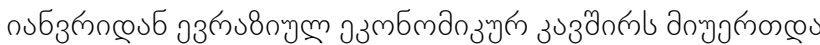

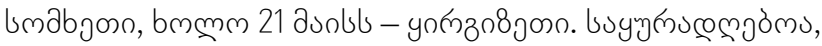
ஙma n⿻

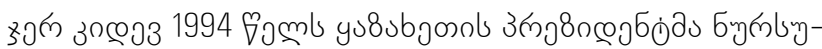

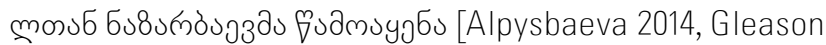
2001, Gleason 2002].

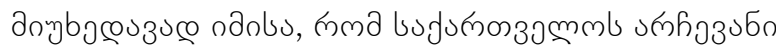

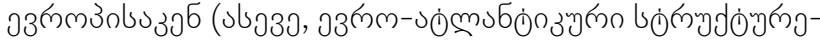

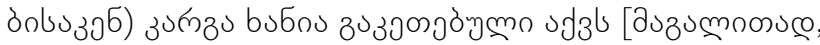

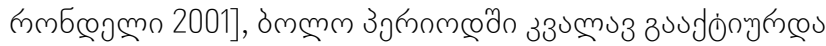

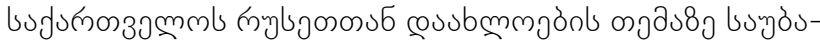

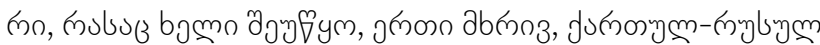

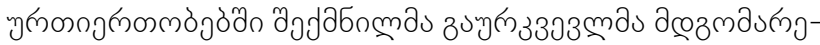

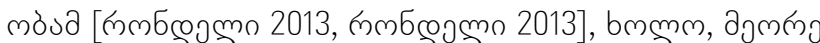

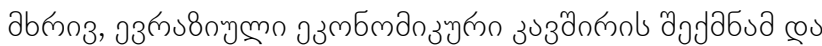

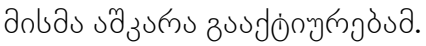

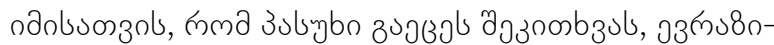

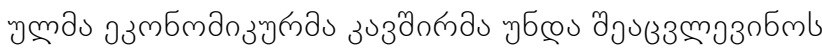

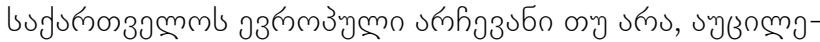

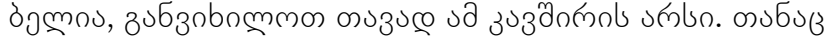

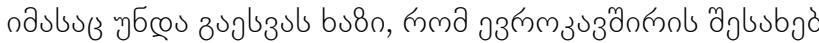

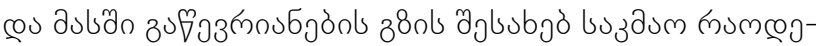

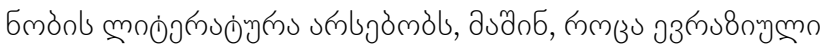

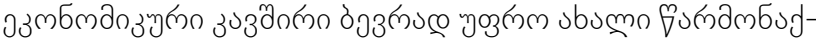

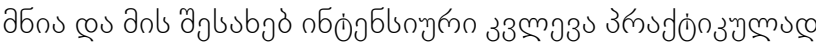

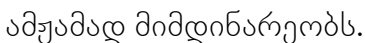

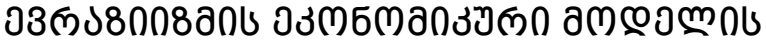

\section{ఇอ৮১bod}

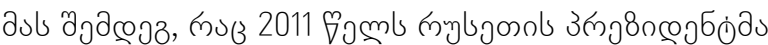

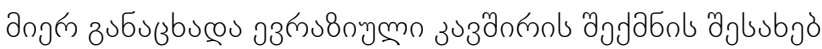

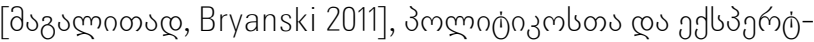

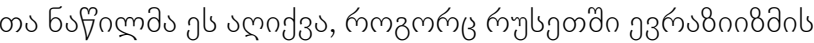

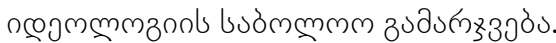

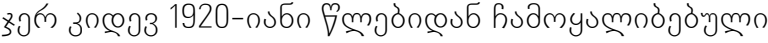

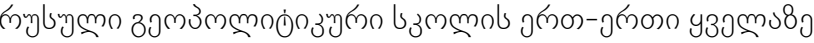

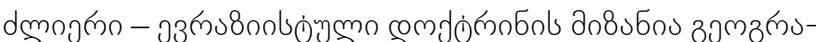

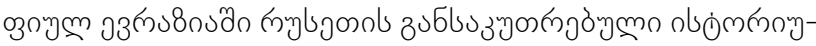

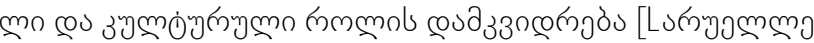

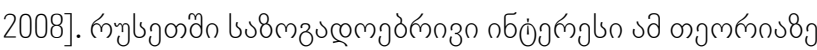

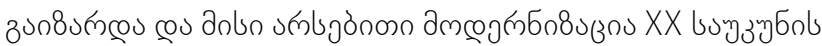

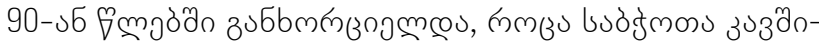

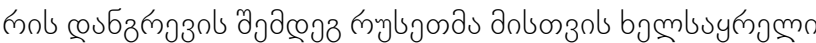

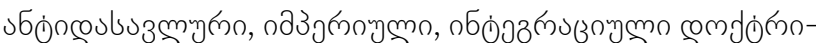
бols [lsmailov \& Papava 2010; Papava 2013] djòбı counfym.

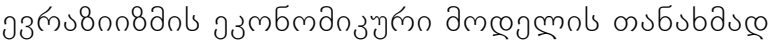




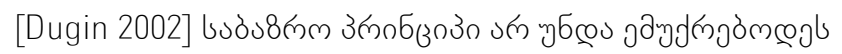

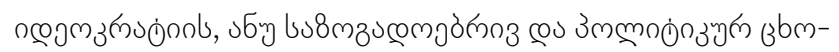

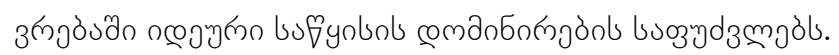

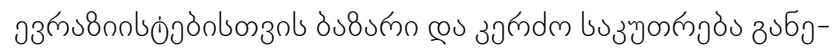

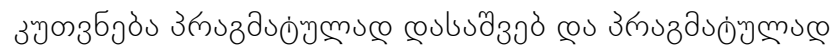

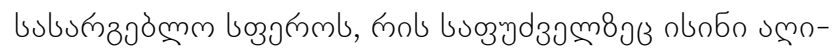

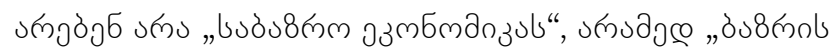

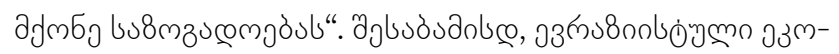

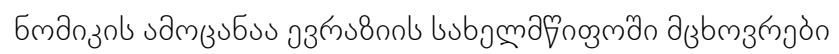

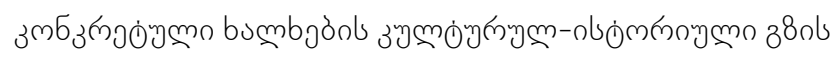

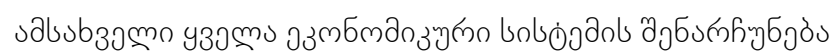

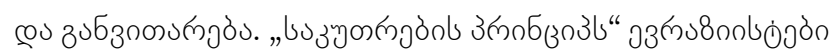

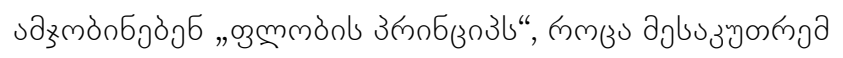

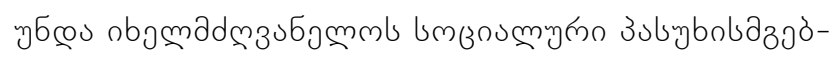

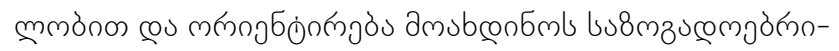

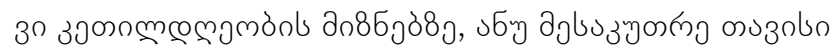

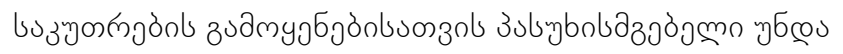

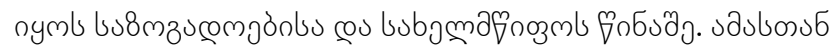

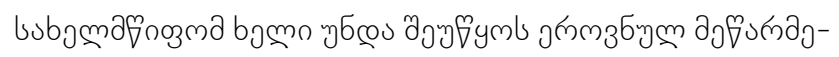

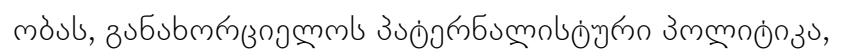

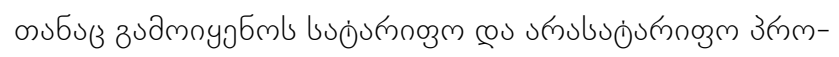

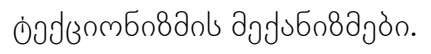

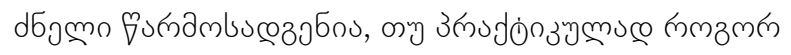

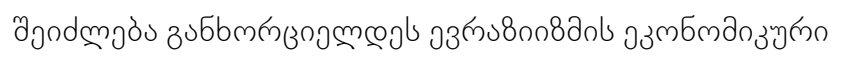

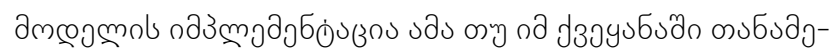

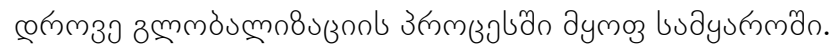

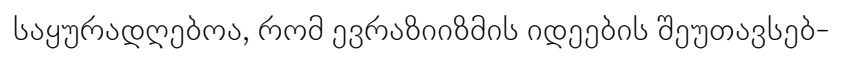

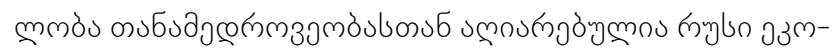

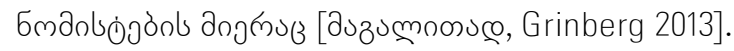

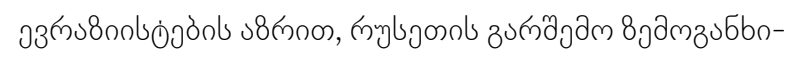

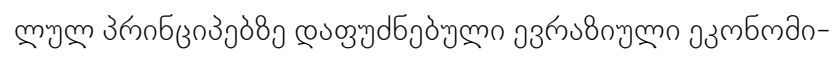

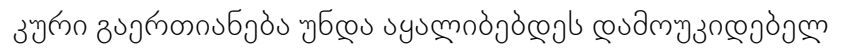

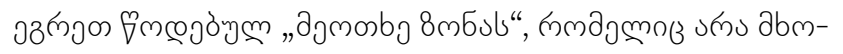

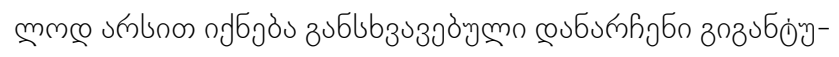

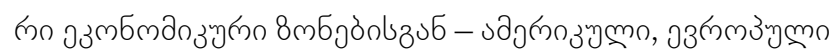

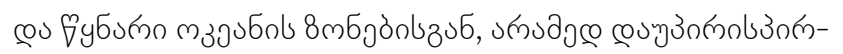

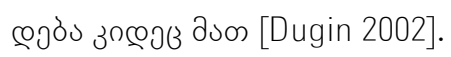

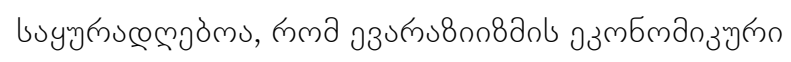

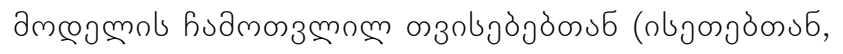

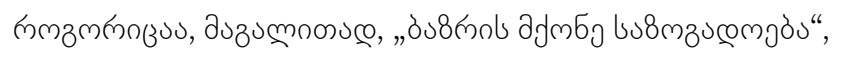

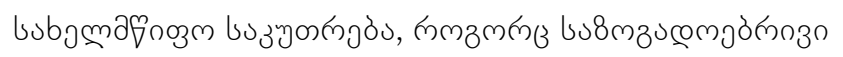

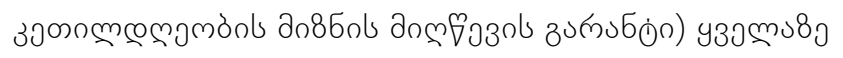

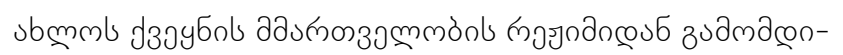

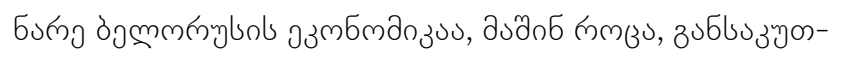

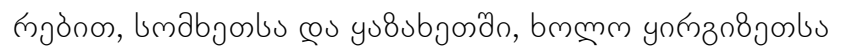

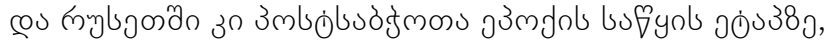

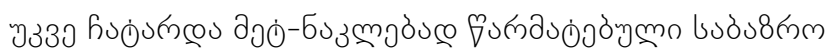

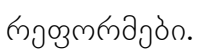

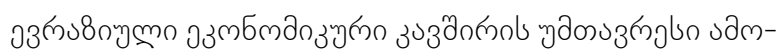

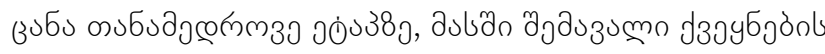

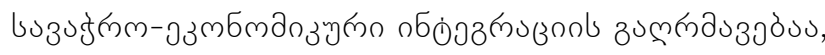

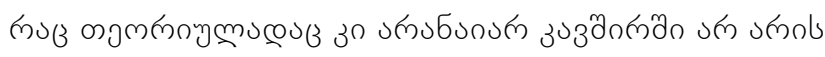

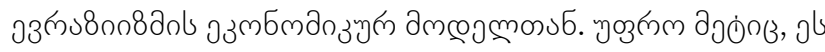

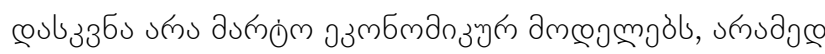

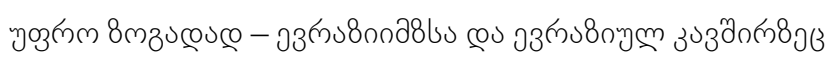

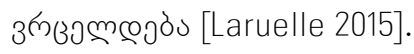

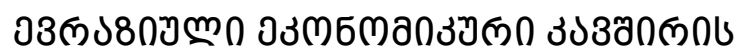

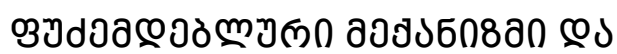

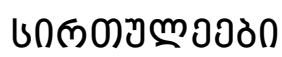

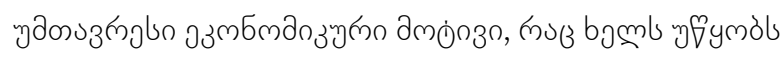

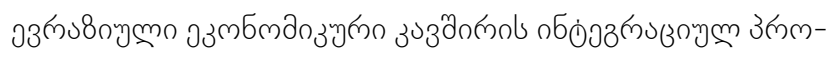

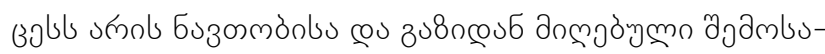

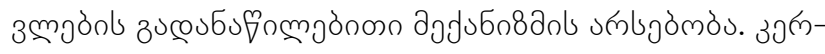

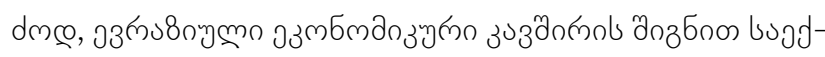

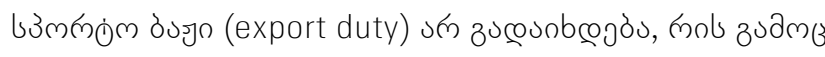

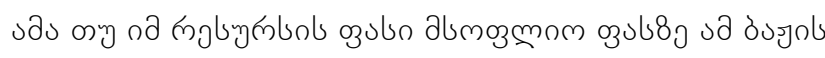

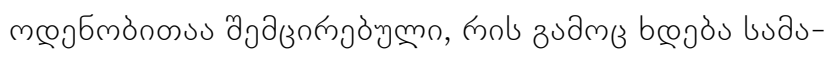

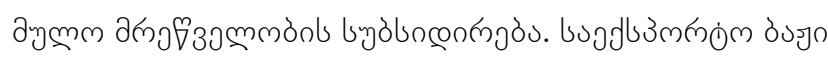

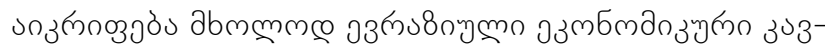

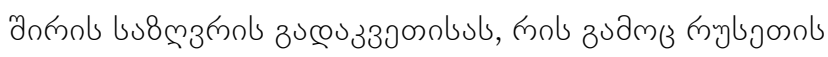

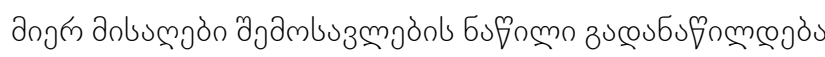

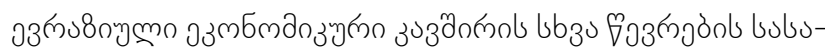

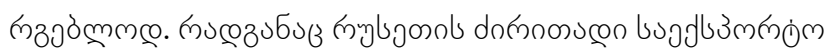

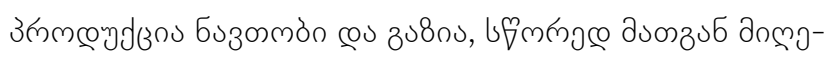

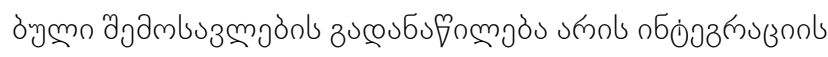

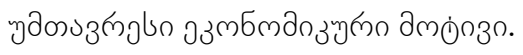

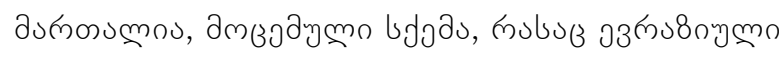

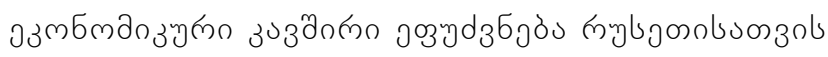

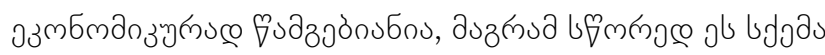

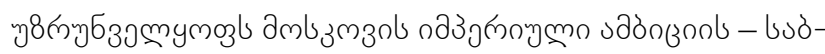

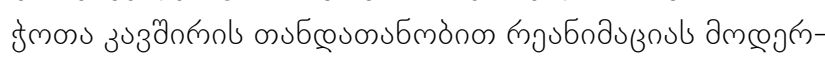

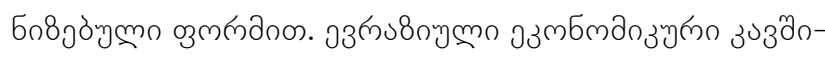

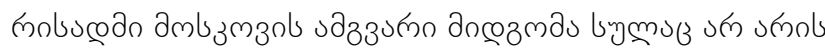

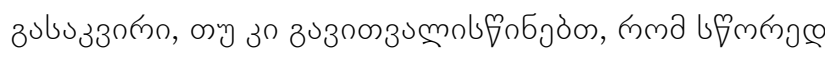

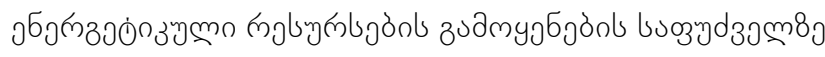

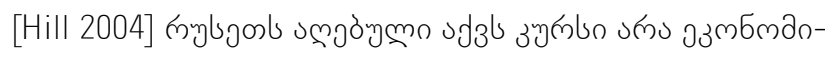

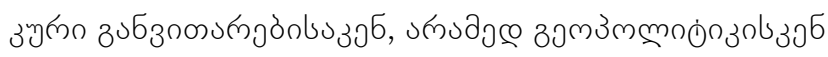
[Appel \& Gelman 2015].

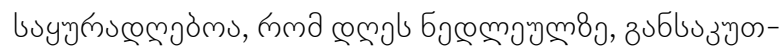




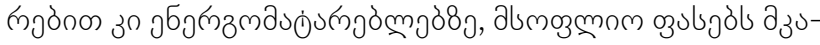

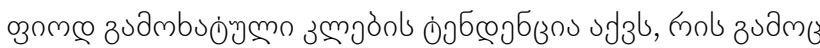

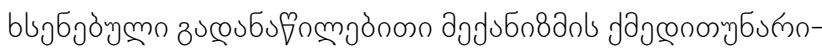

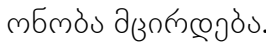

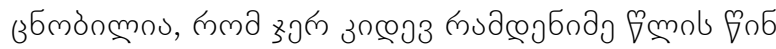
फy

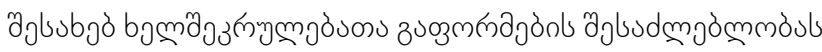

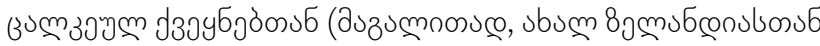

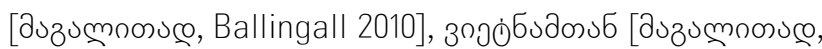
Special Comrades' Russia and Vietnam to Double Trade by 2015], obrnugmons6 [aszummonsco, Keinon 2013], nбcomgonons6 [aszumnoosıc, It's Time for Free Trade Pact with Russia,

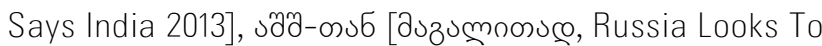

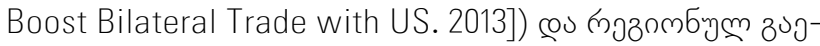
monnuбjòjòonsb (auzumnonuso, ASEAN-onsб [Lisovolik 2010],

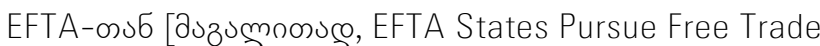
Negotiations with Russia, Belarus and Kazakhstan 2013],

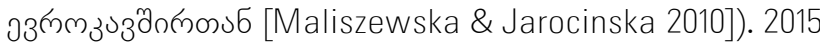

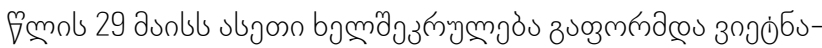

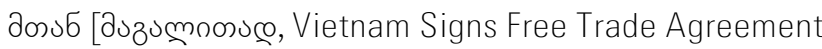

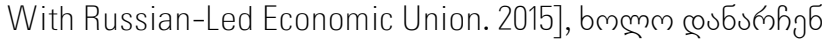

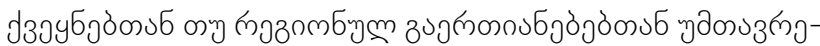

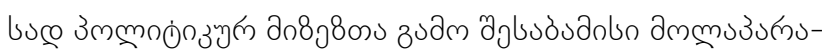

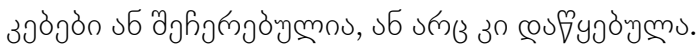

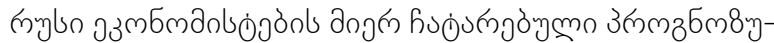

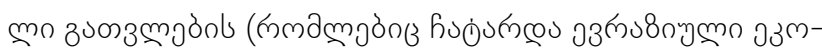

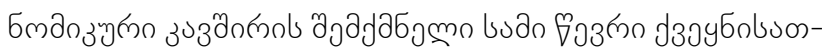

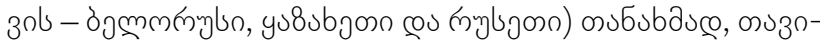

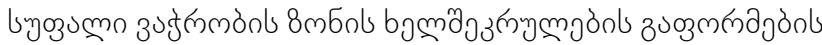

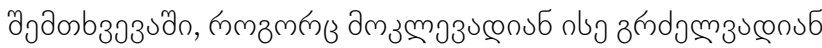

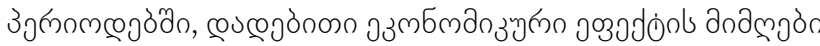

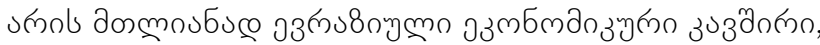

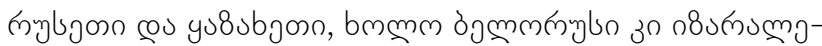

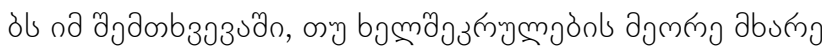

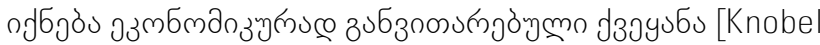

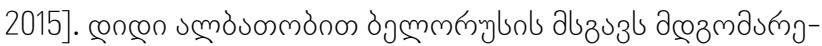

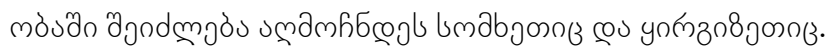

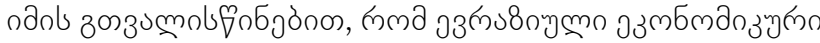

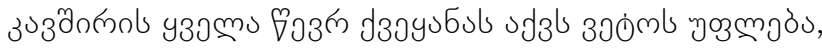

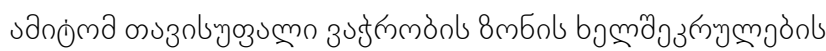

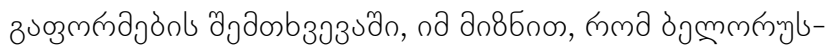

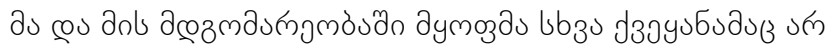
зuдmny

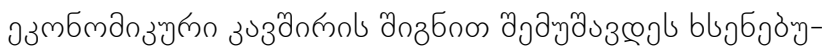

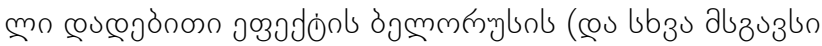

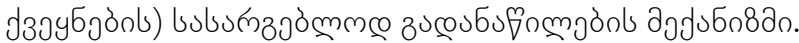

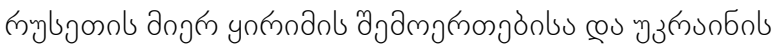

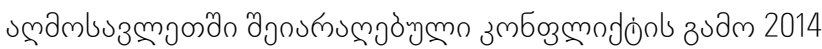

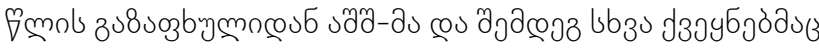

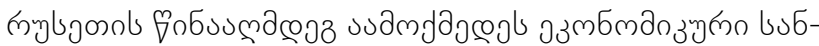

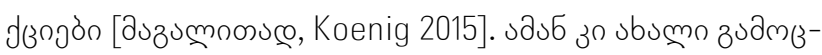

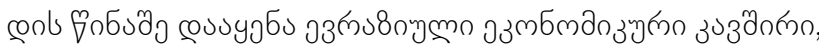

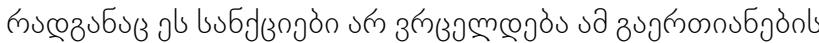
५ьзง

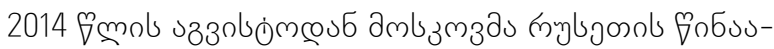

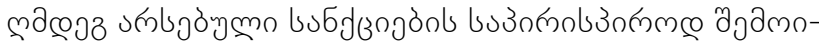

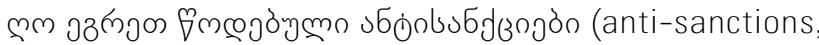
countersanctions) [aszumnoosso, McHugh 2015] - sృjnds-

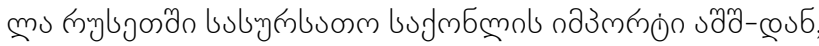

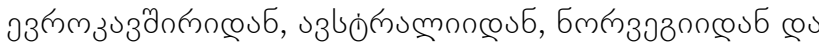

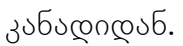

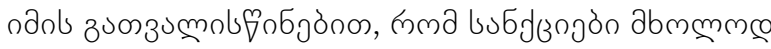

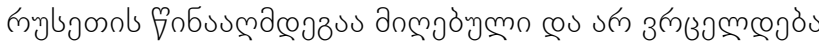

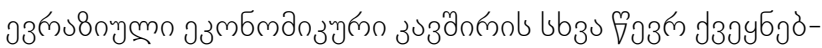

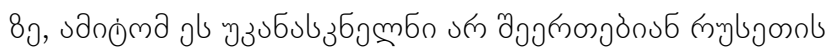

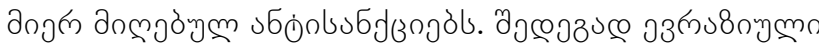

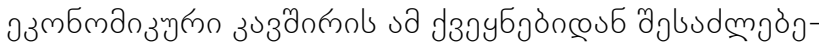

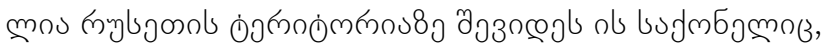

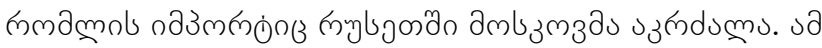

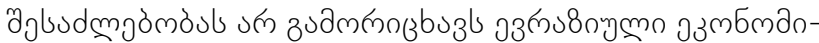

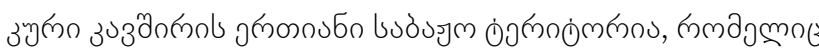

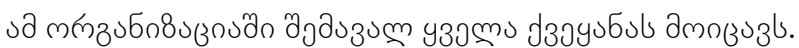

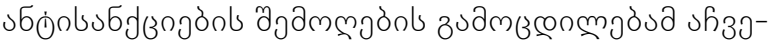

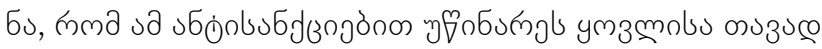

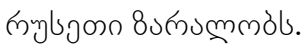

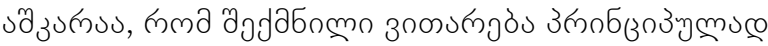

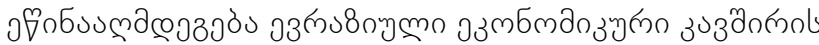

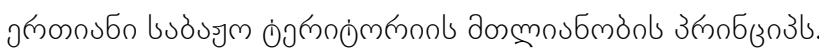

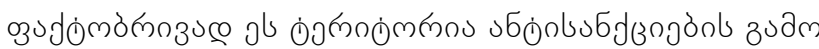

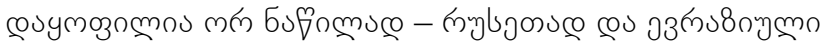

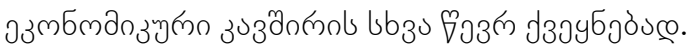

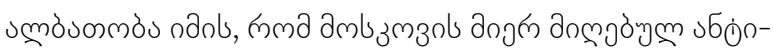

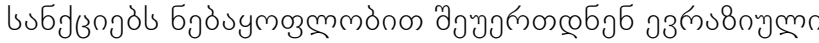

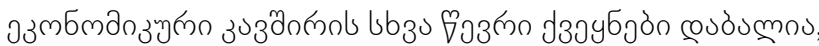

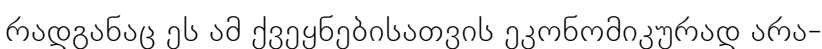

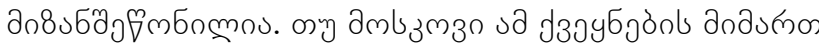

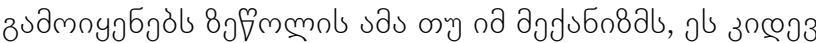

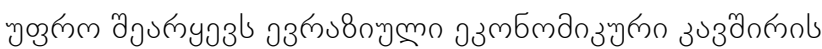

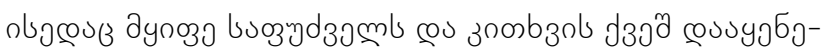

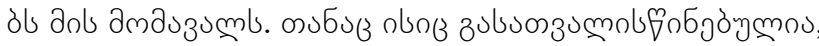




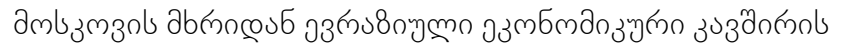

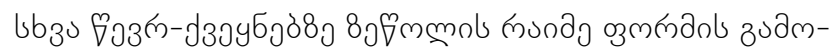

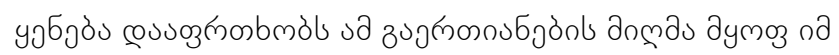

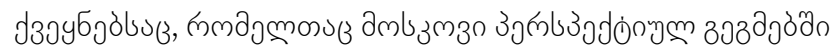

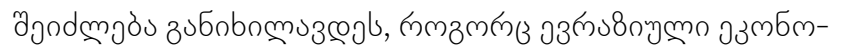

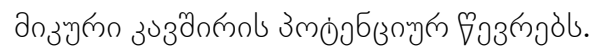

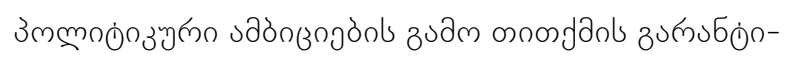

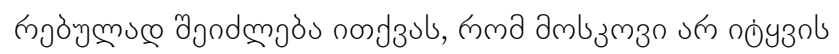

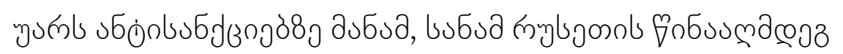

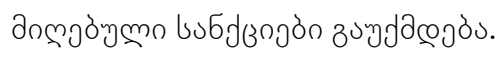

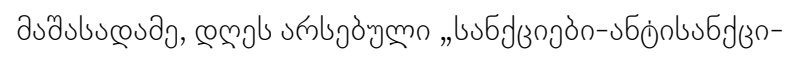

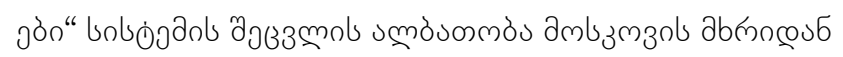

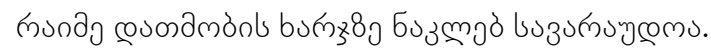

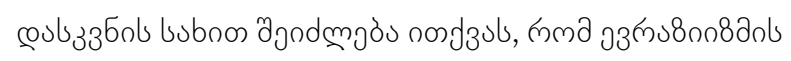

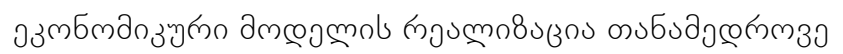

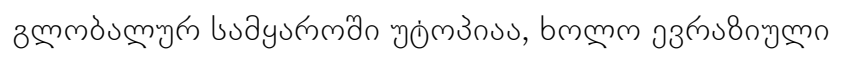

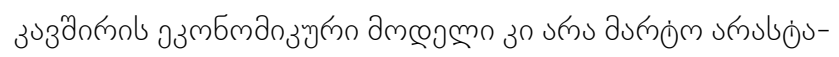

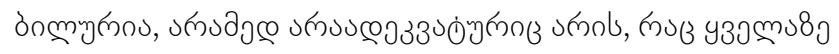

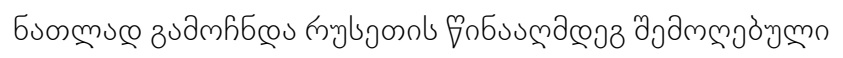

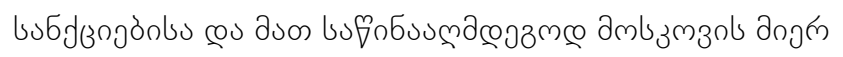

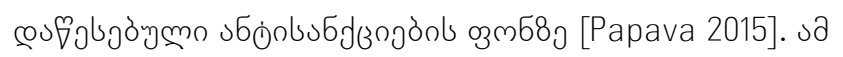

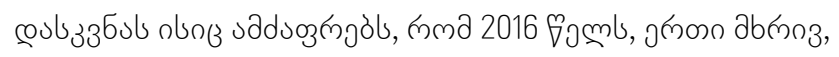

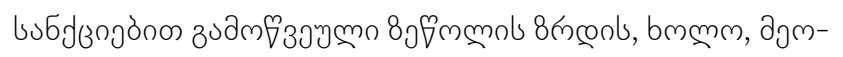

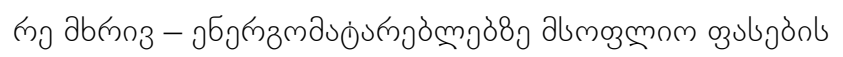

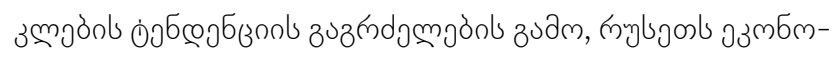

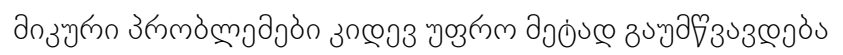
[Dynkin \& Baranovskii 2015].

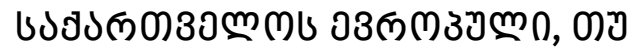

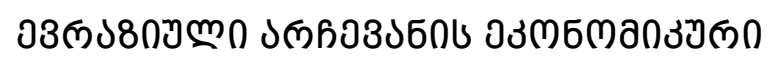

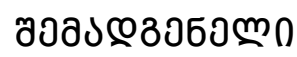

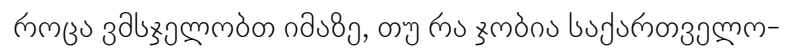

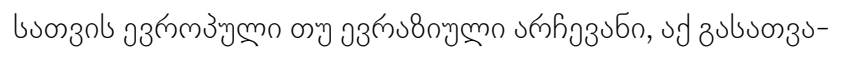

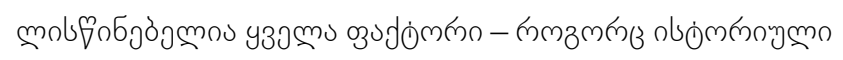

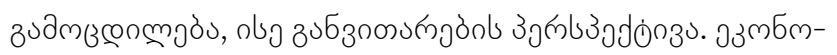

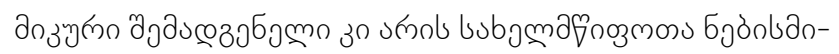

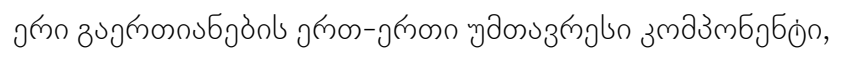

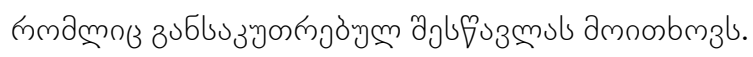

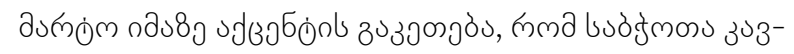

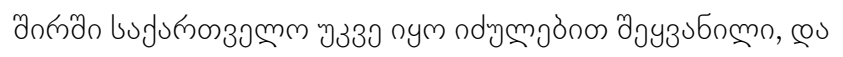

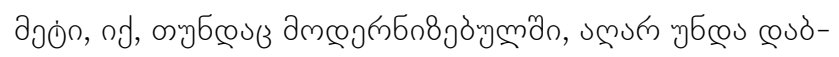

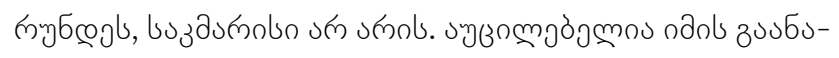

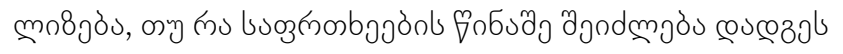

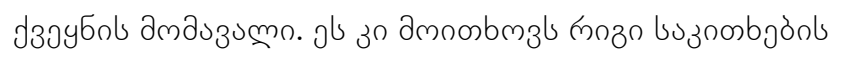

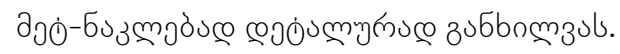

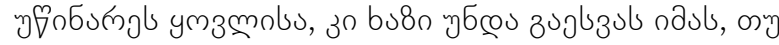

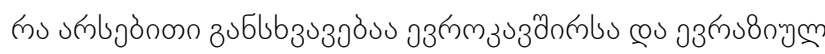

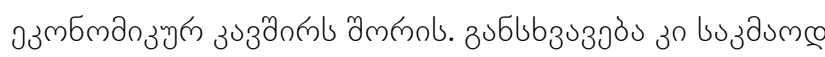

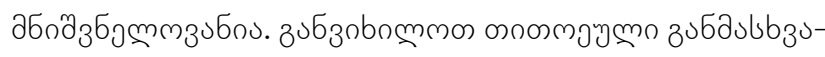
उ०ठ̀े

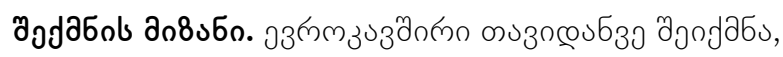

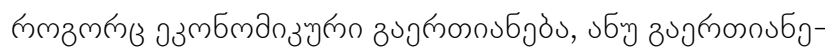

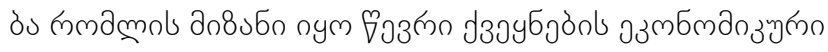

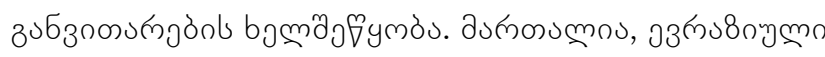

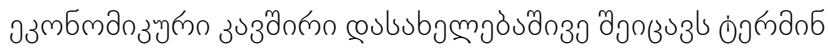

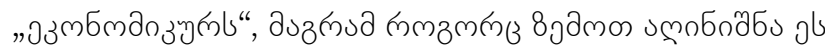

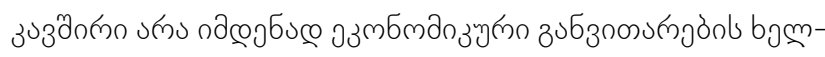

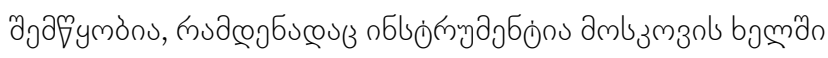

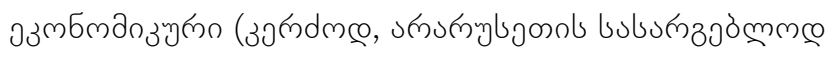

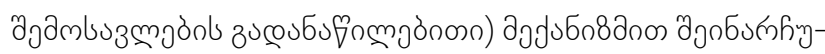

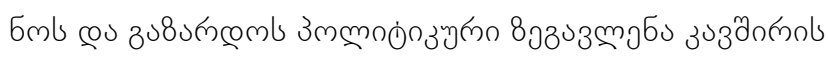

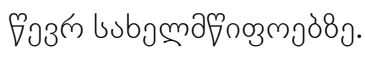

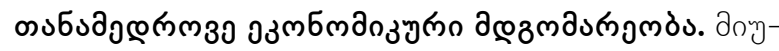

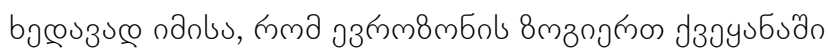

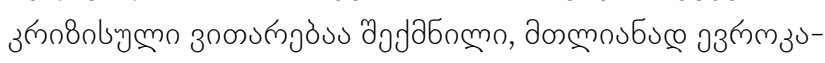

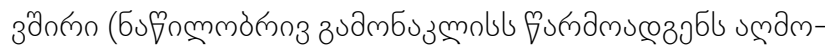

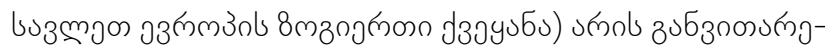

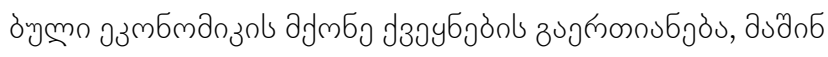

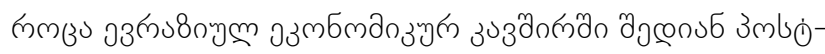

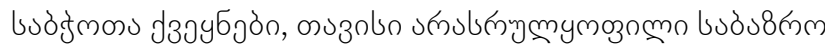

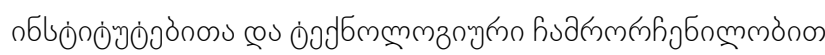

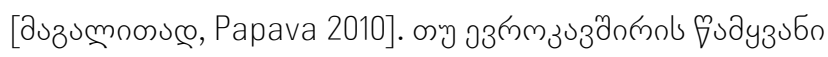

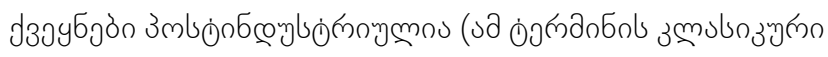

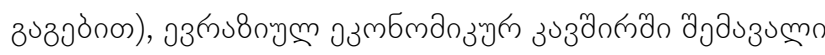

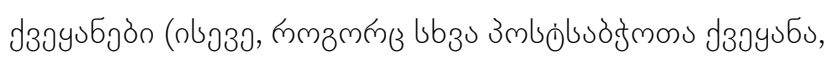

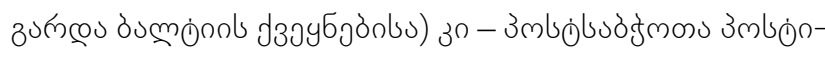

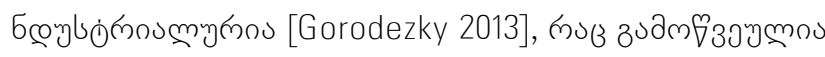

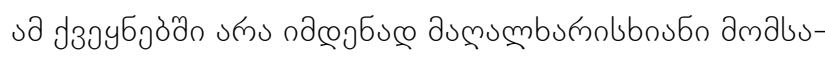

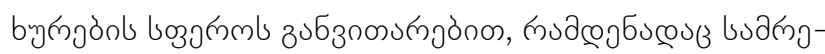

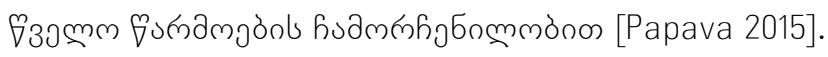

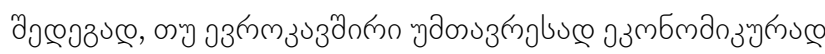

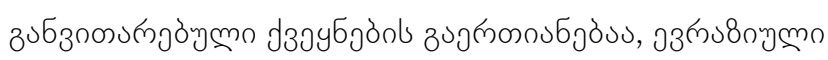

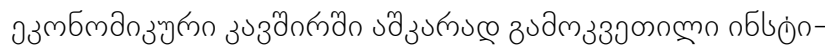

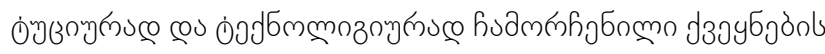

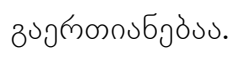

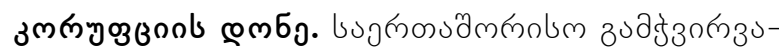

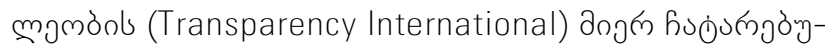

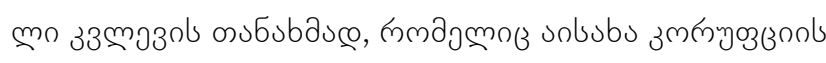

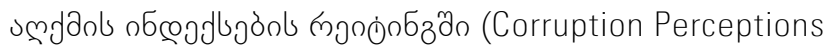
Index ranks) [Corruption Perceptions Index 2014], 2014 


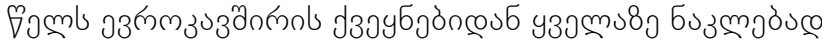

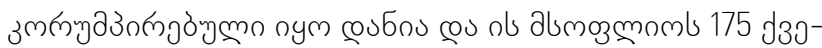

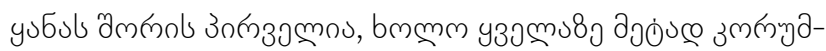

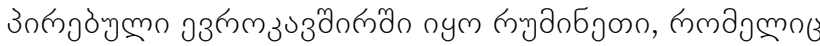

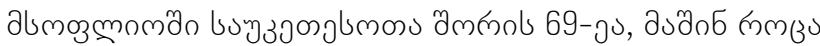

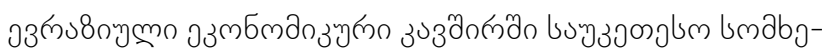

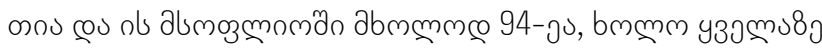

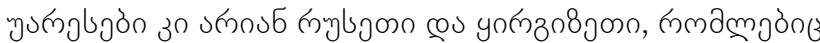

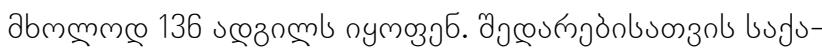

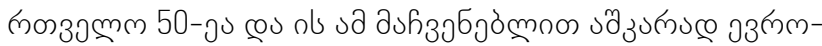

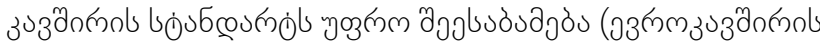

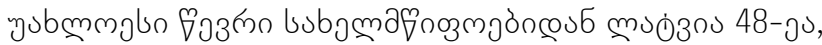

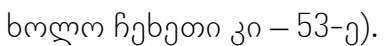

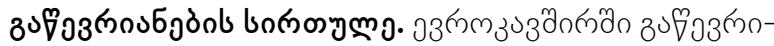

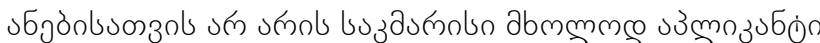

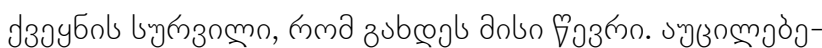

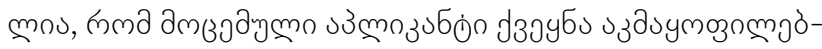

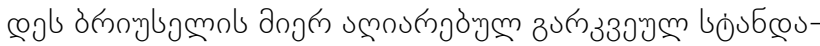

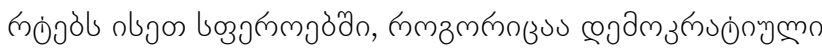

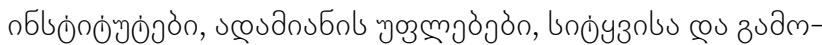

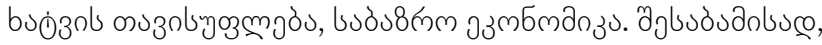

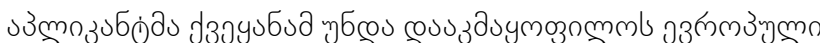

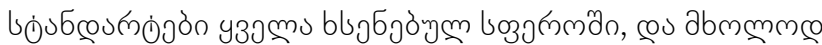

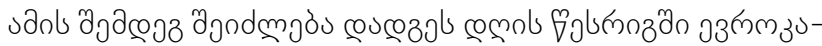

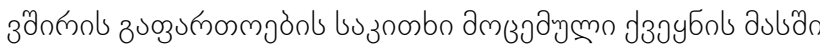

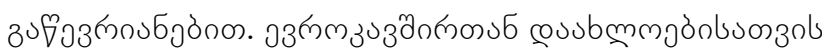

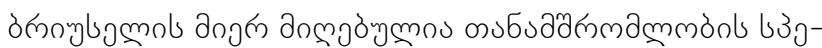

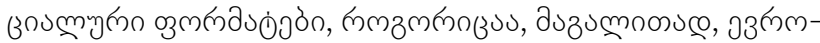

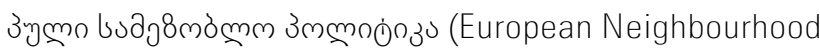

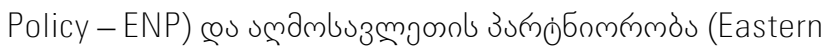

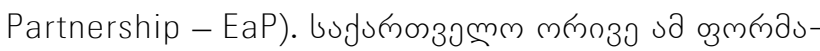

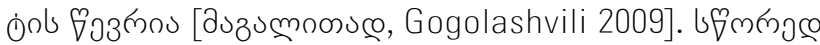

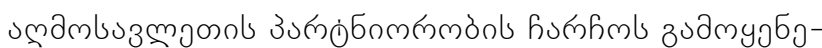

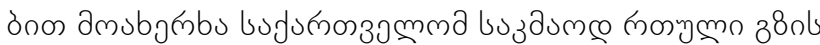

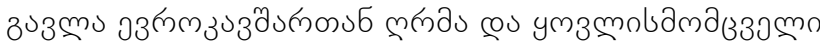

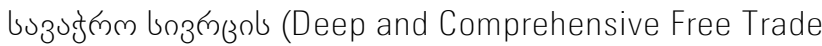

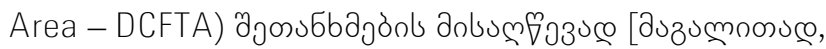

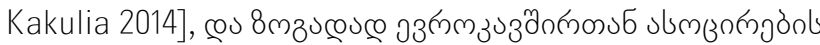

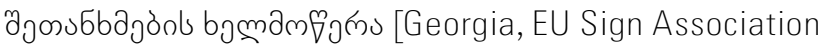
Agreement 2015].

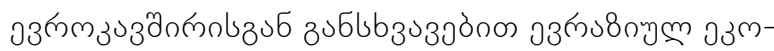

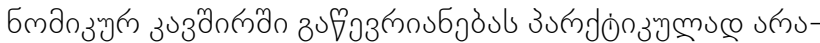

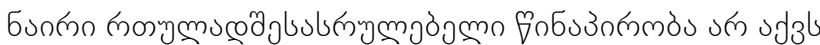
[a১zummonuœe, Evrazii'skii' ekonomicheskii' sojuz 2014],

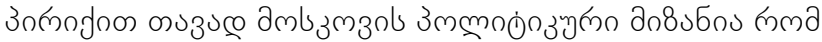

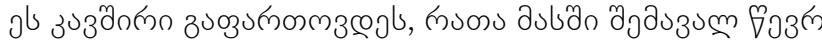

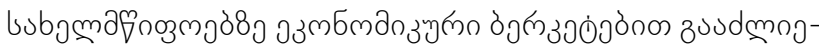

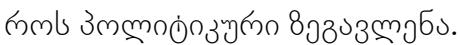

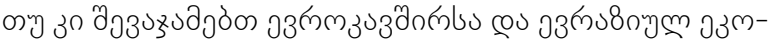

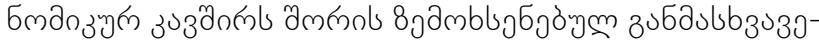

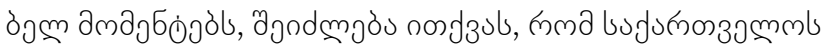

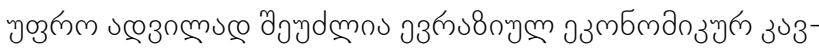

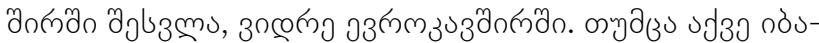

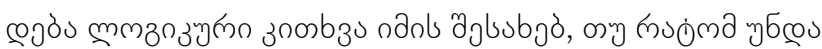

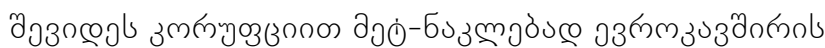

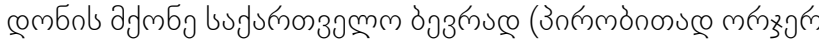

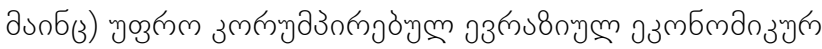

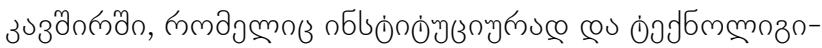

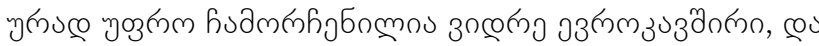

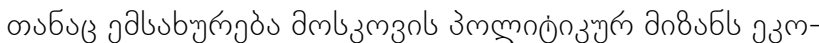

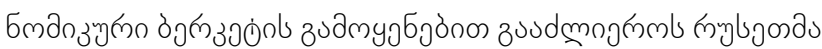

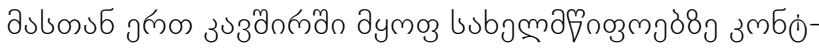

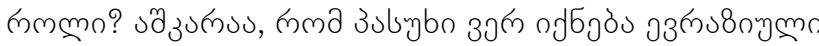

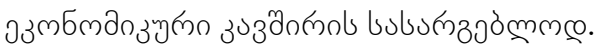

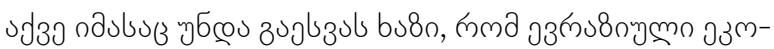

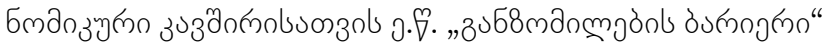

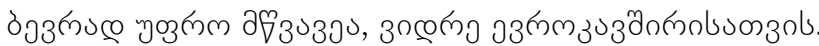

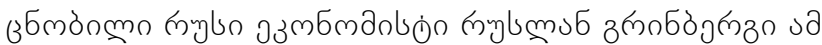

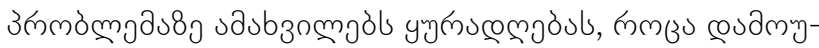

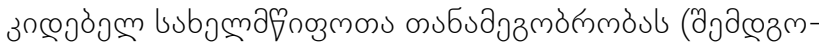

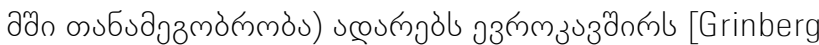

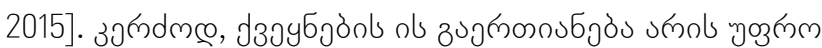

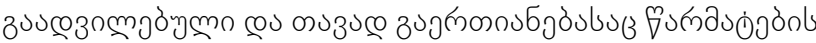

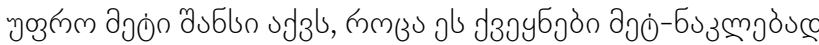

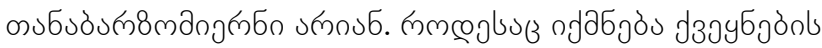
зง grnonn

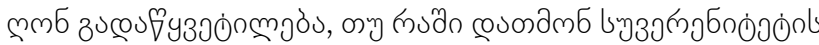

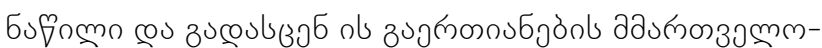

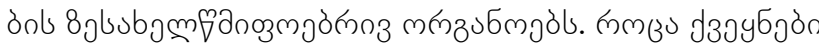

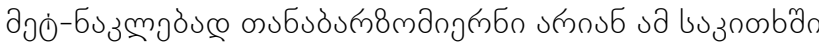

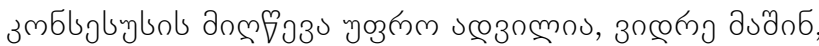

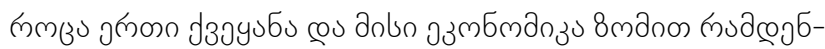

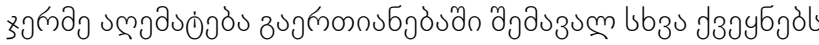
g nonsco u s.

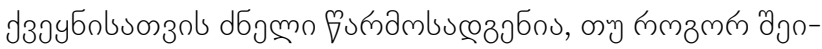

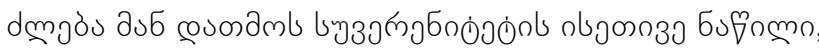

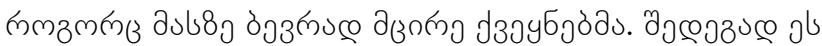

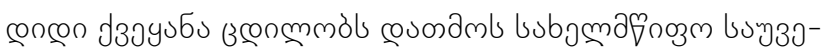

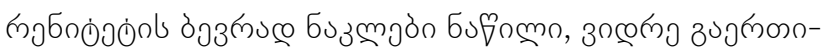

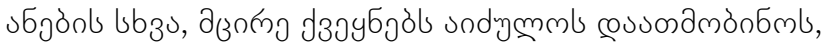




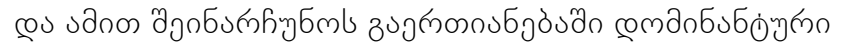

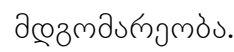

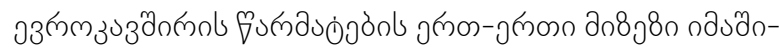

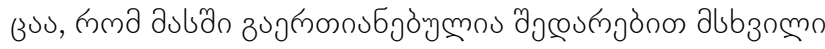

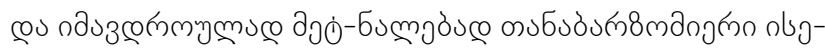

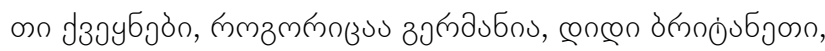

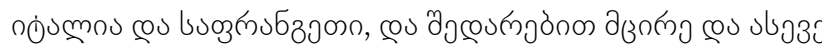

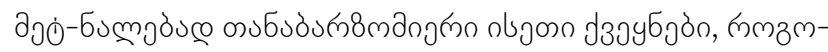

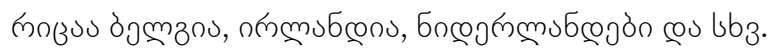

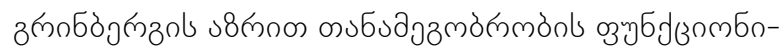

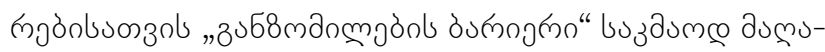
mon nym, nuм

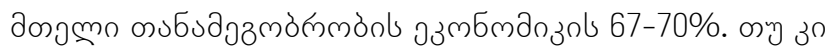

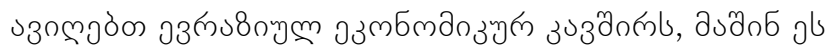

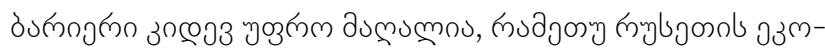

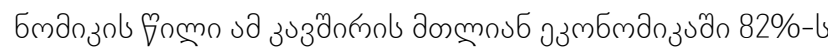

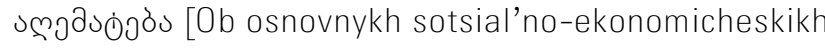
pokazateljakh gosudarstv-chlenov Evraziiskogo ekonomicheskogo sojuza 2015].

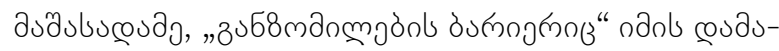

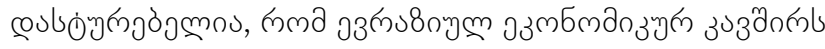

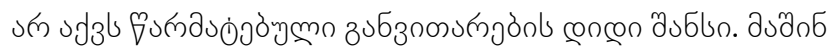

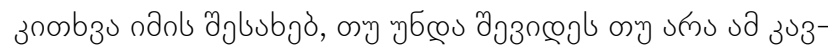

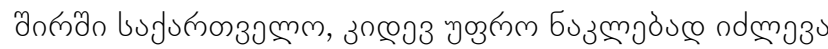

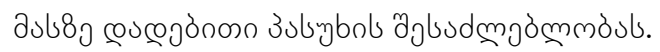

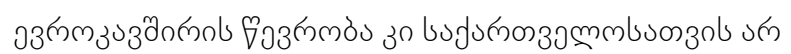

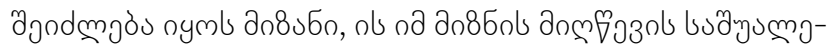

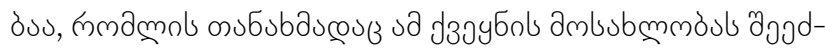

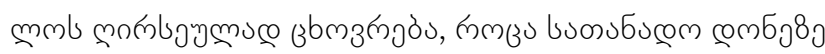

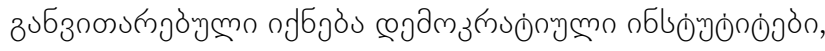

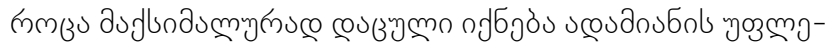

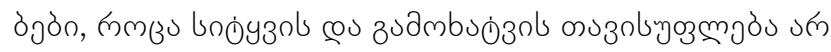

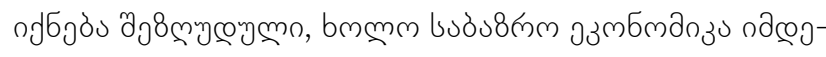
Бsco n

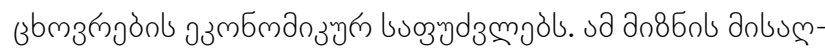

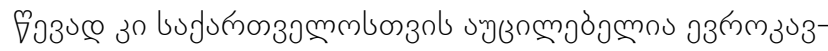

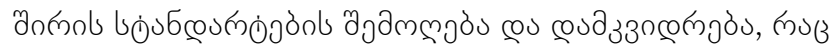

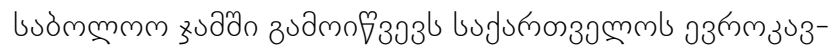
annnosub cousbmmodoll.

\section{cost3365}

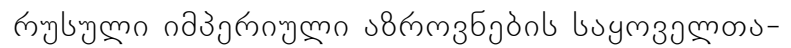

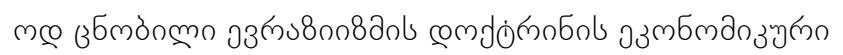

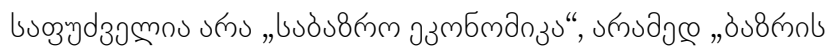

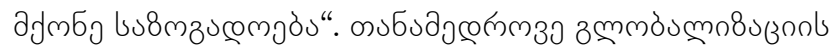

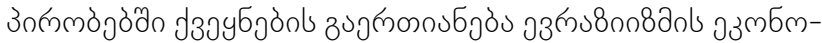

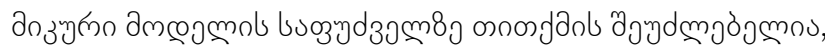

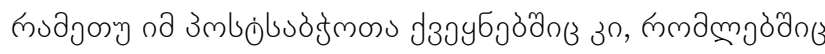

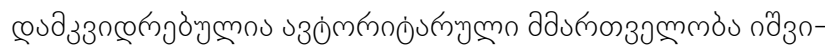

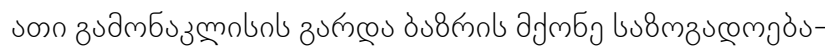

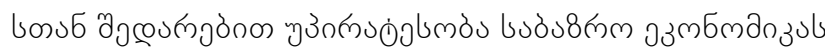
jdmozs.

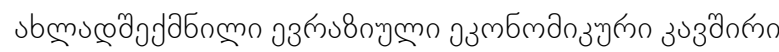

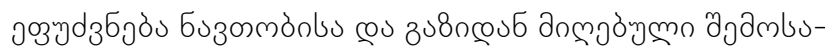

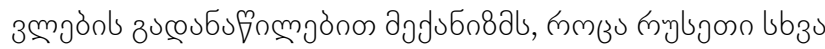

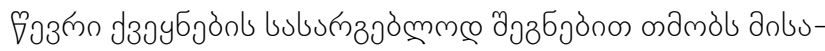

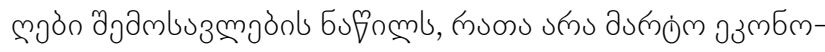

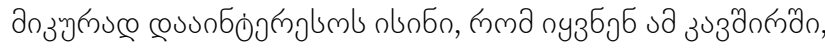

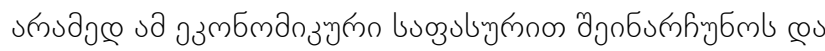

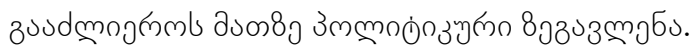

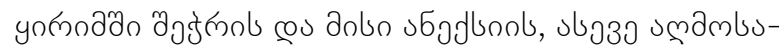

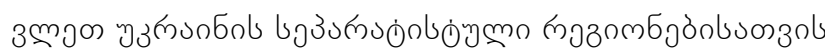

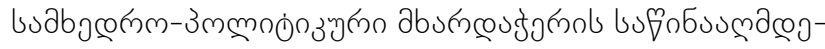

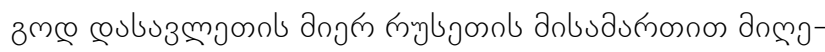

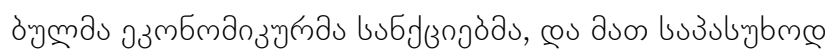

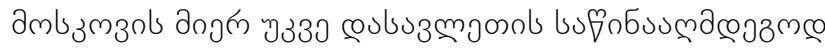

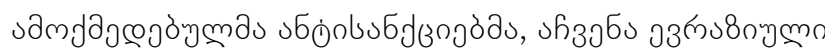

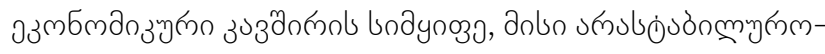

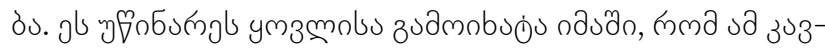

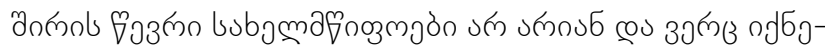

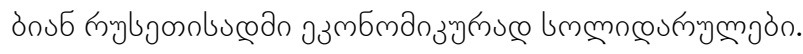

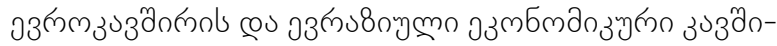

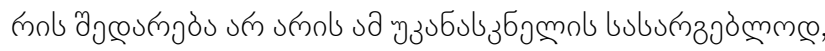

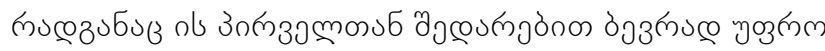

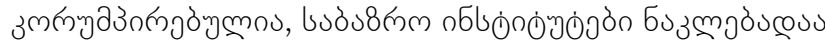

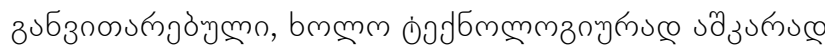

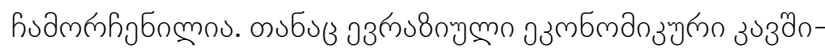

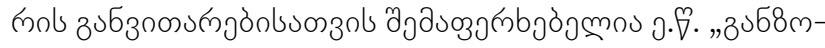

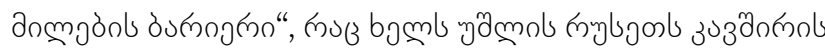

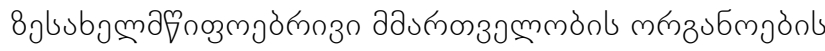

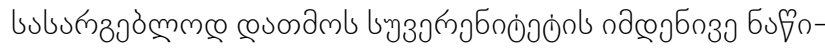

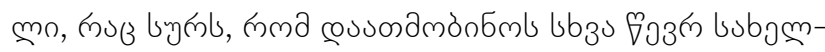
afomgàl.

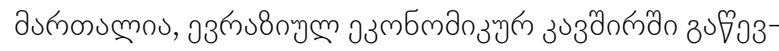

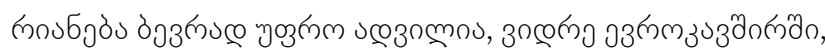

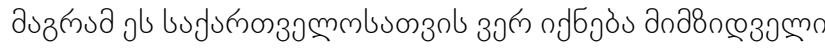

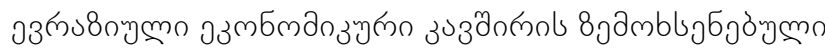

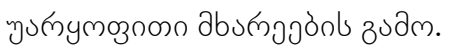




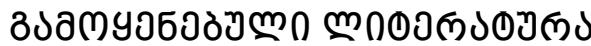

1. Alpysbaeva Zh. (2014). "Nursultan Nazarbaev: Ot idei evrazii'skogo sojuza k perspektivam evrazii'skoi' integratsii [Nursultan Nazarbaev: From the Idea of the Eurasian Union to the Perspectives of the Eurasian Integration]."zakon.kz, May 30, 2014, (In Russian), <http:// www.zakon.kz/4628189-nursultan-nazarbaev-ot-idei. html>; Gleason G. (2001). “Kazakhstan's Nazarbayev Heads New EEC Organization." EurasiaNet, June 17, 2001, <http://www.eurasianet.org/departments/business/ articles/eav061801.shtml>; Gleason G. (2002). “Kazakhstan Positioned as Locomotive of Eurasian Integration.“ EurasiaNet, February 10, 2002, <http://www.eurasianet. org/departments/business/articles/eav021102.shtml>.

2. Rondeli A. (2001). "The Choice of Independent Georgia.“ In The Security of the Caspian Sea Region, G. Chufrin, ed. New York, Oxford University Press, pp. 195-211.

3. Rondeli a. (2013). saqarTvelo-ruseTi: uaryofidan dadebiT gaurkvevlobamde. eqspertis azri \# 3. Tbilisi, saqarTvelos strategiisa da serTaSoriso urTierTobaTa kvlevis fondi. <http://gfsis.org/media/download/library/articles/Expert_ Opinion_GE0_3.pdf>; Rondeli, A. (2013). Georgia-Russia: From Negative to Positive Uncertainty. Expert Opinion No. 3. Tbilisi, Georgian Foundation for Strategic and International Studies, <http://gfsis.org/media/download/ library/articles/Expert_Opinion_ENG_3.pdf>.

4. Bryanski G. (2011). "Russia's Putin says wants to build 'Eurasian Union'."Reuters, October 3, 2011, <http://www. reuters.com/article/us-russia-putin-eurasian-idUS-

TRE7926ZD20111003>; Putin V. “A New Integration Project for Eurasia: The Future in the Making." Permanent Mission of the Russian Federation to the European Union, October 3. <http://www.russianmission.eu/en/news/article-primeminister-vladimir-putin-new-integration-project-eurasiafuture-making-izvestia-3->.

5. Laruelle M. (2008). Russian Eurasianism: An Ideology of Empire. Washington, D.C., Woodrow Wilson Center Press.

6. Ismailov E., Papava V.(2010). Rethinking Central Eurasia. Washington, D.C.: Johns Hopkins University-SAIS, Stockholm: Institute for Security and Development Policy. <http://www.silkroadstudies.org/resources/pdf/ Monographs/2010_MONO_Ismailov-Papava_RethinkingCentral-Eurasia.pdf>; Papava, V. (2013). The Eurasianism of Russian Anti-Westernism and the Concept of 'Central Caucaso-Asia. Russian Politics \& Law, Vol. 51, No. 6, pp. 45-86.

7. Dugin A. (2002). "Ekonomicheskie aspekty neoevraziystva [The Economic Aspects of Neo-Eurasianism], "in Osnovy
Evraziystva [The Fundamental Principles of Eurasianism], A. Dugin, ed. Moscow, Arktogeia-Tsentr, pp. 626-637. (In Russian.)

8. Grinberg R. (2013). Kontury novoiy ekonomiki i shansy evraziyskoiy integratsii [Contours of New Economy and Chances of the Eurasian Integration]. Ekonomisi [Economist], No. 6, pp. 6-11. (In Russian.).

9. Dugin A. (2002). "Ekonomika: chetvertaia zona [The Economy: The Fourth Zone], "in Osnovy Evraziystva [The Fundamental Principles of Eurasianism], A. Dugin, ed. Moscow, Arktogeia-Tsentr, pp. 657-661. (In Russian.).

10. Laruelle M. (2015). Eurasia, Eurasianism, Eurasian Union: Terminological Gaps and Overlaps. PONARS Eurasia Policy Memo, No. 366, July. <http://www.ponarseurasia.org/ sites/default/files/policy-memos-pdf/Pepm366_Laruelle_ July2015.pdf>.

11. Hill F. (2004). Energy Empire: Oil, Gas and Russia's Revival. London, The Foreign Policy Centre. <http://fpc.org.uk/ fsblob/307.pdf>.

12. Appel, H., Gelman, V. (2015). "Revising Russia's Economic Model: The Shift from Development to Geopolitics." PONARS Eurasia Policy Memo, No. 397, November. <http://www.ponarseurasia.org/sites/default/files/policymemos-pdf/Pepm397_Appel-Gelman_Nov2015.pdf>.

13. Ballingall J. (2010). New Zealand-Russia FTA: Another BRIIC in the Wall." NZIER Insight, November 15. <http:// nzier.org.nz/static/media/filer_public/7d/c5/7dc503d6dda7-44b5-b9f1-2e00d613ca6e/nzier_insight_21_-_russia_ fta.pdf $>$.

14. „Special Comrades Russia and Vietnam to Double Trade by 2015." RT Question More, November 12, 2013, <https://www.rt.com/business/russia-vietnam-tradeagreement-580/>.

15. Keinon H. (2013). Israel and Russia to Negotiate FreeTrade Agreement. The Jerusalem Post, December 10. <http://www.jpost.com/Diplomacy-and-Politics/lsraeland-Russia-to-negotiate-free-trade-agreement-334603>.

16. "It's Time for Free Trade Pact with Russia, Says India." The Hindu, April 2, 2013, <http://www.thehindu.com/ news/national/its-time-for-free-trade-pact-withrussia-says-india/article4570802.ece>.

17. Russia Looks To Boost Bilateral Trade with US.“ RT Question More, December 12, 2013, <https://www.rt.com/ business/russia-usa-trade-shuvalov-111/>.

18. Lisovolik Y. (2010). Is a Russia-ASEAN Free Trade Area of Any Use?. International Affairs, Special Issue. <https:// interaffairs.ru/i/pdf_asean/11.pdf>.

19. “EFTA States Pursue Free Trade Negotiations with 
Russia, Belarus and Kazakhstan."EFTA, April 12, 2013, <http://www.efta.int/free-trade/free-trade-news/201304-11-efta-rubeka-8th-rnd-fta-neg>.

20. Maliszewska, M., Jarocinska, E., (2010). Scasny M. Modeling Economic, Social and Environmental Implications of a Free Trade Agreement Between the European Union and The Russian Federation. CASE Network Reports, No. 93. <http://www.case-research.eu/upload/ publikacja_plik/29994065_CNR_93.pdf>..

21. Reuters. "Vietnam Signs Free Trade Agreement With Russian-Led Economic Union. (2015). The Moscow Times, May 29. <http://www.themoscowtimes.com/business/ article/vietnam-signs-free-trade-agreement-withrussian-led-economic-union/522691.html>.

22. Knobel A. (2015). Evraziiskiy ekonomicheskiy sojuz: perspektivy pazvitija i vozmozhnye prepijatstvija [Eurasian Economic Union: Prospects and Challenges for Development], Voprosy ekonomiki [Economic issues]. No. 3, pp. 87-108. (In Russian.)

23. Koenig P.(2015). Russia, The Circus of Economic Sanctions Continues: Washington and Brussels Against Moscow. Global Research, December 24. <http://www. globalresearch.ca/russia-the-circus-of-economic-sanctions-continues-washington-and-brussels-againstmoscow/5497752>.

24. McHugh J. (2015). Russian Sanctions: Putin Signs Decree Ordering All Western Imported Food 'Destroyed'. "International Business Times, July 30. <http://www.ibtimes. com/russian-sanctions-putin-signs-decree-ordering-allwestern-imported-food-destroyed-2031380>.

25. Papava V. (2015). "Economic Models of Eurasianism and the Eurasian Union: Why the Future is Not Optimistic." The Central Asia-Caucasus Analyst, October 29. $<$ http://cacianalyst.org/publications/analytical-articles/ item/13296>.

26. Dynkin, A., Baranovskii, V. (2015). eds. Rossija i mir: 2016. Ekonomika i vneshnjaja politika. Ezhegodmyi prognoz [Russia and the World: 2016. Annual Forecast: Economy and Foreign Policy]. Moscow, IMEMO RAN. <http://www. imemo.ru/files/File/ru/publ/2015/2015_024.pdf>.

27. Papava V. (2010). The Economic Challenges of the Black Sea Region: the Global Financial Crisis and Energy Sector Cooperation." Southeast European and Black Sea Studies, Vo. 10, No. 3, pp. 361-371.

28. Gorodezky A.(2013). Antikrizisnoe regulirivanie i modeli postkommunisticheskogo razvitija Rossii [Crisis Manage- ment and the Post-Crisis Development Model for Russia]. Ekonomika Ukrainy [Economy of the Ukraine]. No. 5, pp. 66-74. (In Russian.)

29. Papava V. (2015). "Necroeconomics of Post-Soviet PostIndustrialism and the Model of Economic Development of Georgia and Russia.“ Journal of Business and Economics, 2015, Volume 6, No. 5, pp. 976-983, <http://www. academicstar.us/UploadFile/Picture/2015-7/20157313847837. $\mathrm{pdf}>$.

30. “Corruption Perceptions Index 2014: Results." Transparancy International, <http://www.transparency.org/ cpi2014>.

31. Gogolashvili K. (2009). "The EU and Georgia: The Choice is in the Context." In Mkrtchyan T., Huseynov T., Gogolashvili K. The European Union and the South Caucasus. Three Perspectives on the Future of the European Project from the Caucasus. Europe in Dialogue 2009/01. Gütersloh, Bertelsmann Stiftung 2009, <http://eeas.europa.eu/ delegations/georgia/documents/virtual_library/30_xcms_ bst_dms_en.pdf>.

32. Kakulia M.(2014). “Georgia's Experiences on Developing Trade and Trade Policy Relations with the European Union.“East European Studies, No. 5, pp. 137-160, <http:// vki.hu/files/download_772.html>.

33. “Georgia, EU Sign Association Agreement." (2015). Civil.Ge, June, 27, 2015, <http://www.civil.ge/eng/article. php?id=27417>.

34. Evrazii'skii' ekonomicheskii' sojuz (2014).. Voprosy i otvety. Tsifry i fakty [Eurasian Economic Union. Questions and Answers. Figures and Facts]. Moscow, Evrazii'skaja ekonomicheskaja komissija [Eurasian Economic Commission], 2014, <http://www.eurasiancommission. org/ru/Documents/eaes_voposy_otvety.pdf>.

35. Grinberg R. (2015). “Kontury novoi' ekonomiki i shansy evraziyskoi' integratsii [Contours of New Economy and Chances of the Eurasian Integration].“

36. “Ob osnovnykh sotsial'no-ekonomicheskikh pokazateljakh gosudarstv-chlenov Evraziiskogo ekonomicheskogo sojuza [On the Main Social and Economic Indicators of the Member States of the Eurasian Economic Union].“(2015). Evraziiskaja ekonomicheskaja komissija [Eurasian Economic Commission]. January-November, 2015, <http://www.eurasiancommission.org/ru/act/ integr_i_makroec/dep_stat/econstat/Documents/Indicators201511.pdf 


\title{
Global challenges and rational choice of Georgia
}

\author{
https://doi.org/10.35945/gb.2016.02.001
}

\author{
Vladimer Papava \\ Doctor of Economic Sciences, Professor of Ivane Javakhishvili Tbilisi State \\ University, Academician
}

Key words: European Union, Eurasian Economic Union, the dimension of the barrier

$G$ eorgia today stands at crossroads between two alternatives: to continue rapprochement with the European Union (EU) on a basis of the Association Agreement (and to ultimately pursue membership through a lengthy, drawn-out process), or to join the Eurasian Economic Union (EAEU), a much simpler prospect. Georgia has long made clear that it favors engagement with Europe and EuroAtlantic institutions; however, discussion of Georgia's rapprochement with Russia is becoming more and more topical as a result of uncertainty in modern Georgia-Russia relations and the establishment of the EEAU. This essay clarifies the main differences between the EU and EAEU in the wider context of Georgia's future. The EAEU started operations in 2015 and at present includes five membercountries - Armenia, Belarus, Kazakhstan, Kyrgyzstan, and Russia. Georgia is located between two member states Armenia and Russia - which creates new challenges for Georgia's development. Georgia and the EU signed the EUGeorgia Association Agreement in June 2014. Despite this agreement, talks on Georgia's rapprochement with Russia have recently reignited, largely sparked by the establishment of the EAEU. When analyzing the suitability of these two organization for Georgia, it is important to consider the essential differences between them:

- The EU was initially set up as an economic union, with the aim of promoting the economic develop- ment of its member states. Although the EAEU contains the term "economic" in its title, this union is not so much a means of economic development as it is a mechanism through which Moscow seeks to maintain and increase its political influence on the member states.

- The EU is, with the partial exception of some Eastern European member states, an association of developed economies, while the EAEU is comprised solely of underdeveloped post-Soviet economies deficient in their market institutions and lagging behind global standards in technology.

- Transparency International's Corruption Perceptions Index demonstrates an essential disparity between the EU and EAEU on the issue of corruption. The most corrupt state in the EU according to this ranking is Bulgaria (75th of 175), while the least corrupt in the EAEU is Belarus (79th). For comparison, Georgia ranks 44th.

- For a country to join the EU, it must meet certain standards set by Brussels in areas such as democratic institutions, human rights, freedom of speech and expression, and market economy. Furthermore, only after an applicant country has met European standards in the above areas is the issue of formal membership placed on the agenda. In order to 
encourage rapprochement with the EU, Brussels has adopted special formats of cooperation - for instance, the European Neighborhood Policy (ENP) instrument and the Eastern Partnership (EaP). Georgia is a participant in both formats. It is through the application of the EaP framework that Georgia has managed to successfully traverse the rather difficult path towards the entry into force of the Deep and Comprehensive Free Trade Area (DCFTA) and the Association Agreement. Unlike the EU, the EAEU has virtually no complex preconditions for membership. On the contrary, Moscow's aim is to expand the union in order to increase its political influence on member-states via economic leverage, with no concern for economic and political standards such as those emphasized by the EU.

- When the essential differences between the EU and the EAEU are summed up, it can be concluded that Georgia can more easily attain membership in the latter than in the former. However, this evokes a separate question: why would Georgia, a country with a more or less EU-level standard of corruption, enter into the much more corrupt EAEU, which lags behind the EU in institutional and technological terms, and serves Moscow's political objective of strengthening Russian control over the member states? The answer, of course, is that it would not be in Georgia's interest to pursue EAEU membership.

Moreover, it is important to emphasize that the socalled "commensurability barrier" for the EAEU is much more significant than for the EU. Ruslan Greenberg, a Russian economist, outlines this issue through the comparison of the Commonwealth of Independent States (CIS) and the EU Greenberg shows that an alliance of countries is streamlined and possesses a higher chance of success when the commensurables (sizes) of the member countries are more or less comparable. When an alliance of countries is formed, the states concerned should make a decision on the areas where they are ready to relinquish part of their sovereignty in favor of the supranational governing bodies of the association. When the commensurability of the countries is more or less analogous, reaching consensus on this matter is easier than when one country and its economy are several times larger in size than those of all the other constituents of the union put together. In this case, the largest country finds it difficult to imagine how it can be expected to yield a share of its sovereignty equivalent to that of much smaller states. As a result, this large country attempts to relinquish far less of its state sovereignty than it obligates the other smaller member states to surrender, thereby maintaining a dominant position in the association.

One of the reasons of the EU's success is also the fact that it consolidates relatively large and simultaneously commensurably more or less homogeneous countries, such as Germany, Great Britain (before the implementation of Brexit), Italy and France, and relatively small but commensurably comparable countries, such as Belgium, Ireland, the Netherlands, and others.

According to Greenberg, the "commensurability barrier" for the CIS was rather high, since the Russian economy accounted for 67-70 percent of the entire economy of the CIS. This barrier is even larger in the EAEU, as Russia's constitutes over $82 \%$ of the entire economy of the union. The issue of, the "commensurability barrier" is a further indication that the EAEU does not have a high chance of success and an additional. 